\title{
Type Synthesis and Typical Application of 1T2R-Type Parallel Robotic Mechanisms
}

\author{
Fugui Xie, ${ }^{1,2}$ Xin-Jun Liu, ${ }^{1,3}$ and Tiemin $\mathrm{Li}^{1,3}$ \\ ${ }^{1}$ Department of Mechanical Engineering, Tsinghua University, Beijing 100084, China \\ ${ }^{2}$ Department of Precision Instruments, Tsinghua University, Beijing 100084, China \\ ${ }^{3}$ Beijing Key Lab of Precision/Ultra-Precision Manufacturing Equipments and Control, Tsinghua University, Beijing 100084, China
}

Correspondence should be addressed to Fugui Xie; xiefg@mail.tsinghua.edu.cn

Received 2 July 2013; Accepted 20 August 2013

Academic Editor: Marcelo Moreira Cavalcanti

Copyright (c) 2013 Fugui Xie et al. This is an open access article distributed under the Creative Commons Attribution License, which permits unrestricted use, distribution, and reproduction in any medium, provided the original work is properly cited.

\begin{abstract}
This paper focuses on the 1T2R-type (T: translational DOF; R: rotational DOF) parallel robotic mechanisms (PKMs) and discusses their type synthesis and typical application in five-axis machine tools. Based on Grassmann line geometry and atlas method, a systematic method dealing with the type synthesis of lower mobility PKMs is introduced. The Blanding rules and generalized Blanding rules, which are the criterions in realizing the mutual conversion between the freedom-space atlas and the constraintspace atlas, are summarized and discussed in detail. Thereafter, the entire procedure of the type synthesis is presented, and the type synthesis of 1T2R PKMs is carried out. Based on the synthesis results, a five-axis hybrid mechanism is proposed and a machine tool is developed consequently. The type synthesis method presented in this paper is intuitive and concise and can be used in the type synthesis of other lower mobility PKMs.
\end{abstract}

\section{Introduction}

Parallel kinematic mechanisms (PKMs) $[1,2]$ are characterized by multi-closed-loop structure [3], which is distinctly different from the open-loop structure of the traditional mechanisms, namely, serial kinematic mechanisms (SKMs). This distinct structure makes the PKMs have the features of high rigidity, low moving inertia, high dynamic performance [4], and high accuracy potential at the expense of strong coupling in terms of kinematics. For such a reason, PKMs have been studied intensively by the researchers from both academia and industry [5]. Gough-Stewart platform [6] is the first proposed and also the most famous six-degreeof-freedom (DOF) parallel structure; it has been extensively investigated from then on. The PKMs' unique merits mentioned above make them suitable for the application of machine tools, especially in processing parts with complicate and freeform surfaces. Some engineering practices also indicate that machine tools based on PKMs outperform those based on SKMs in surface finish and geometric accuracy when machining hardened materials [7]. In this field, some machine tools based on fully parallel mechanisms are developed, such as Variax [7], Hexapod [8], Metrom, and others. Variax and Hexapod are based on the Gough-Stewart platform, and both of them need six-axis computer numerical control (CNC) and have relatively small workspace, especially the limited rotational capacity. Metrom is based on a 5DOF fully parallel mechanism, and its rotational capacity can achieve $90^{\circ}$. This is a great advantage in the field.

In comparison, machine tools based on hybrid mechanisms, which combine the advantages of both PKMs and SKMs, have been extensively applied in industry and gradually became the development trend of high-speed, highaccuracy, and high-flexibility machine tools. In the hybrid structure, the parallel module is used to realize the machining performance of high-speed, high-accuracy, and highflexibility; meanwhile, the serial module is used to achieve large workspace. This structure can decrease the number of joints, mobility inefficiency [9], and structural complexity. It is a good compromise and better solution for the machining application. The successful application of Tricept machines, Verne machine [10], Ecospeed series machines, and others. has demonstrated this fact. All of the mentioned machines belong to the category of $3 \mathrm{~T} 2 \mathrm{R}$ ( $\mathrm{T}$ : translational DOF; 
R: rotational DOF) and are composed of a 3-DOF parallel module and a 2-DOF serial module. Of note is that the 3DOF PKMs are one of the most important classes of lower mobility PKMs and adopted most often in the five-axis hybrid mechanisms. This is due to the fact that 3-DOF PKMs have the features of simple kinematics and weak coupling, and these factors make them easier to use as machining modules.

In processing aircraft parts characterized by thin wall or parts with complex and freeform surfaces, the rotational flexibility and capacity of five-axis machine tools can significantly influence the machining capability. Therefore, they should be fully taken into consideration in the mechanism configuration design stage. Traditionally, the high rotational flexibility and capacity is achieved by serially adding an A/C articulated tool head or an A/B rotary table. However, the rotating cutter of the machine tool based on this architecture sometimes scratches the finished surface during setup, and this has set back the further improvement of machining speed. To explore better solutions, lower mobility PKMs with two rotational DOFs are attractive to researchers. Among them, the 1T2R PKMs [11-13] are of intriguing interest recently as the development of hybrid mechanisms. The 1T2R PKMs, which have flexible rotation capacity, can realize the function of $\mathrm{A} / \mathrm{B}$ articulated tool head and without the drawbacks of this kind of serial tool heads [14]. So, this kind of mechanisms is very suitable to be as a machining module in a five-axis hybrid machine tool. Focusing on the 1T2R PKMs, this paper will mainly discuss their type synthesis and typical application in five-axis hybrid machine tools.

To create more novel mechanisms with useful functions is a significant and challenging issue in the domain of PKMs $[15,16]$, especially those with lower mobility. As is well known, topological configuration is fundamental and decisive to the comprehensive property of a mechanism and will inevitably influence the performance of the developed machine tool on the basis of this mechanism. Therefore, the determination of topological configuration (i.e., type synthesis) of a mechanism is the first critical step for the successful design of a machine tool. According to the requirement for DOFs of a mechanism, type synthesis can determine the topological configuration based on a certain theory. The most commonly used method in the field is topological graphs (TG) [17]. A lot of work has been contributed to this area and has promoted the development of lower mobility PKMs. Many effective theories or methods dealing with type synthesis have been proposed, such as the methods based on screw theory [18-21], displacement group theory [22-25] using Lie Group Algebra, single-opened-chains units $[26,27], G_{F}$ set [28], and theory of linear transformation and geometrical analysis [29]. All of the theories are very effective, and lots of novel mechanisms have been generated and proposed based on these theories. Using these theories to guide the practice of type synthesis, one should grasp the relatively complicated procedures and the profound mathematical theory first. This is a disadvantage for their widespread application. This paper will try to do some contributions to this area and introduce a more concise and intuitive method for the type synthesis of lower mobility PKMs. Type synthesis of 1T2R PKMs and the corresponding results based on the method will be presented, and an application example will be introduced sequentially.

The remainder of this paper is organized as follows. The next section introduces the type synthesis method based on Grassmann Line Geometry and Atlas Method. Some basic criterions and rules are presented, and the diagram of type synthesis process is given. Thereafter, the type synthesis of 1T2R PKMs is carried out and the results are listed. Section 3 discusses the typical application of the 1T2R PKMs generated in the last section. A five-axis hybrid mechanism is proposed and a prototype is developed consequently. Conclusions are given in Section 4.

\section{Type Synthesis Based on Grassmann Line Geometry and Atlas Method}

2.1. Grassmann Line Geometry and Atlas Method. Grassmann Line Geometry is a mathematical theory investigating the geometrical features of spatial line-clusters. Based on this theory, the linear dependence of line vectors can be classified and expressed by their dimensions. This kind of description makes Grassmann Line Geometry easier to integrate with the relevant research of mechanism theory, especially the freedom and constraint. In this field, Grassmann Line Geometry has been applied to mechanisms' singularity analysis [30-32] and flexible mechanisms' design $[33,34]$. Some line-clusters under different dimension are listed in Table 1.

Based on Grassmann Line Geometry and the physical meanings given to the spatial line-clusters, the mechanisms' motion and constraint can be expressed by line graphs, which are composed of linear vectors or couples. In general, line graphs contain four basic elements as shown in Table 2. In which red color denotes constraint, blue color denotes freedom, line without arrows denotes vector and line with double arrows denotes couple. These elements can be used to constitute a line graph, which can express an $n$-dimensional $(n \leq 6)$ freedom space or constraint space. The process using freedom- or constraint-space line graphs to describe mechanisms' motion or constraint is called Atlas Method. This method is intuitive and concise, and it also has clear and definite physical meaning. Therefore, the qualitative analysis of mechanisms' motion and constraint can be carried out in an intuitive way.

This paper focuses on the type synthesis of 1T2R parallel mechanisms; so, some typical three-dimensional freedom spaces and corresponding physical meanings are presented in Table 3. If the color of the atlases in Table 3 is changed into red, these atlases can be used to describe three-dimensional force or couple constraints according to the definitions in Table 2. Based on Grassmann Line Geometry and Atlas Method, this paper brings in dual principle of motion and constraint to deal with the type synthesis of 1T2R parallel mechanisms. The synthesis procedure and results will be presented in the following sections.

2.2. Basic Rules and Procedure of the Type Synthesis. Using Grassmann Line Geometry and Atlas Method to carry out 
TABLE 1: The typical line-clusters of Grassmann Line Geometry.

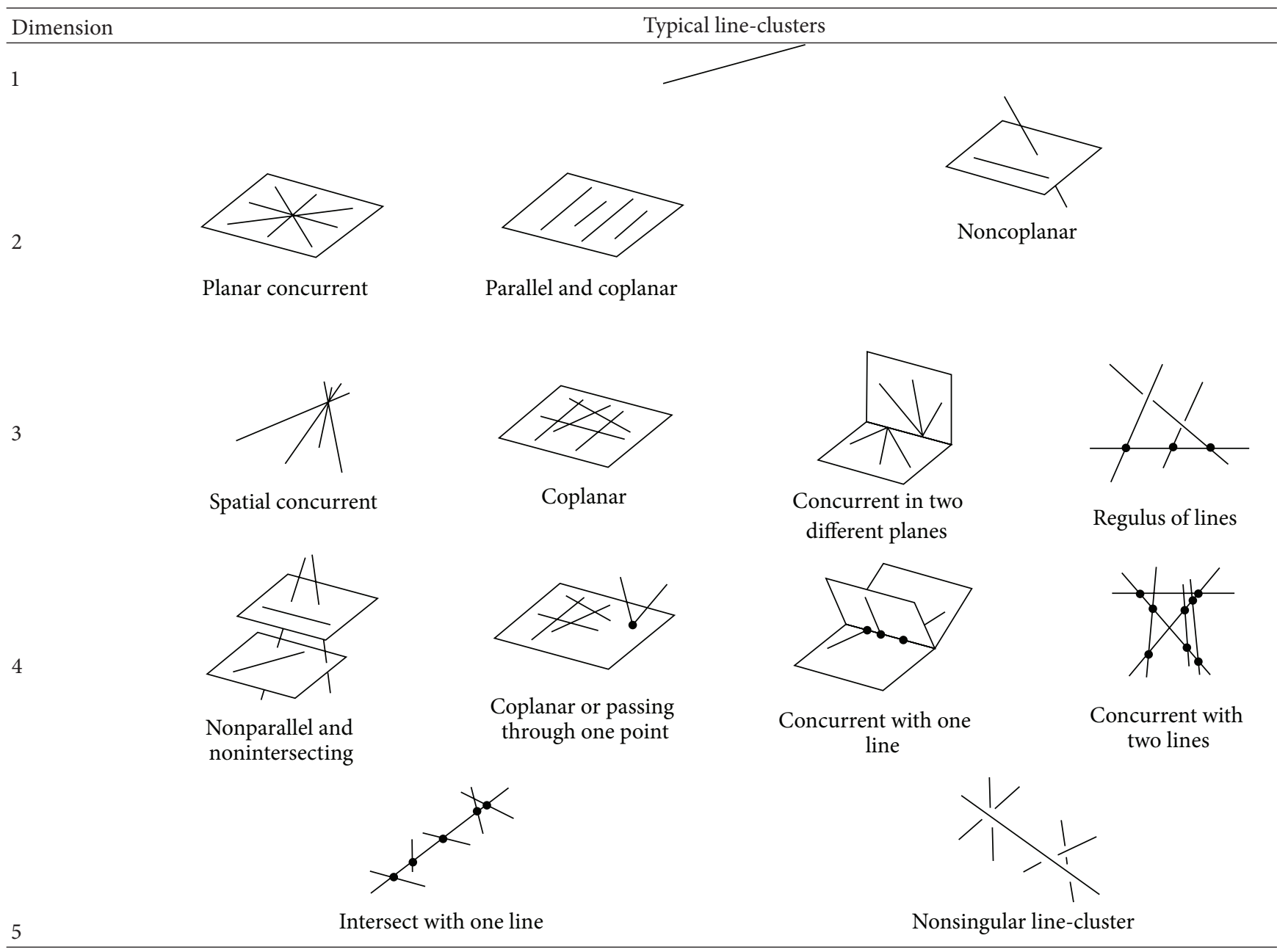

TABLE 2: Basic elements in line graph and their meanings.

\begin{tabular}{ccc}
\hline Basic element & Mathematic meaning & Physical meaning \\
\hline & Vector & Rotational DOF \\
& Vector & Constraint force \\
& Couple & Translational DOF \\
& Couple & Constraint couple \\
\hline
\end{tabular}

type synthesis, some basic criterions and rules are indispensable. In this section, the criterions of Grassmann Line Geometry and Blanding rules are summarized; thereafter, the technological process of the type synthesis is introduced in detail.

\subsubsection{Basic Criterions of Grassmann Line Geometry. Using} Grassmann Line Geometry to express the freedoms and constraints of a mechanism, the dimensions (i.e., the numbers of independent lines or couples) of the corresponding line clusters should be identified first. The following criterions lay the foundation for this process:

(1) there are at most three independent lines in a plane;

(2) there are only two independent parallel lines in a plane;

(3) there are only three independent parallel lines among all of the spatially parallel lines;

(4) there is only one independent couple among all of the parallel couples in space;

(5) there are only two independent lines among all of the coplanar and concurrent lines;

(6) there are only three independent lines among all of the concurrent lines in space;

(7) for two sets concurrent lines (or one set concurrent lines and one set parallel lines) in two different planes, there are only three independent lines if the intersections lie in the intersecting line of the two planes;

(8) there are at most five independent lines in two or more planes that intersect at one line; 
TABLE 3: Some three-dimensional freedom spaces under the description of Atlas Method.

Physical meaning
$\begin{aligned} & \text { Two-dimensional rotations and } \\ & \text { one-dimensional translation }\end{aligned}$
$\begin{aligned} & \text { Two-dimensional translations and } \\ & \text { one-dimensional rotation }\end{aligned}$
Three-dimensional translations
Three-dimensional rotations

(9) there are at most five independent lines in two or more parallel planes.

Generally, a line graph contains a lot of lines and couples. The number of independent lines or couples, that is, the dimension of the line graph, can be identified according to the criterions listed above. In the description of freedom and constraint of mechanism, there exists dual relationship between the freedom lines and the constraint lines. Therefore, a close relationship exists between the corresponding line graphs, and the following rules will be very helpful in investigating this relationship.

2.2.2. Blanding Rules. Blanding proposed a basic rule that reflects the dual relationship between the constraint and freedom, this rule can be summarized as follows.

Assuming that a line graph contains $n$ independent (nonredundant) lines, then the corresponding dual graph contains (6- $n$ ) independent (nonredundant) lines, and each line in line graph intersects with all lines in the dual graph.

According to this rule, the dual constraint graph can be uniquely identified when the freedom graph is given, and vice versa.

Investing the physical meanings of motion or force to the dual graphs, a generalized Blanding rule [33] can be summarized as follows:

(1) the axes of rotational DOFs of a mechanism intersect with the lines of all constraint forces;

(2) the axes of translational DOFs of a mechanism are orthogonal to the lines of all constraint forces;

(3) the axes of rotational DOFs of a mechanism are orthogonal to the axes of all constraint couples;

(4) the axes of translational DOFs of a mechanism and the axes of all constraint couples can be in any direction.

This rule is very helpful to identify the dual relationship between the freedom lines and constraint lines in a concise and intuitional way and realize the mutual conversion between freedom-space atlas and constraint-space atlas. Then, the analysis with respect to DOFs and constraints of a mechanism can be carried out based on the two kinds of atlases.

2.2.3. Technological Process of the Type Synthesis. Based on the above ideas, the type synthesis process based on Grassmann Line Geometry and Atlas Method can be summarized as follows.

(1) Determine freedom space according to the DOFs of the mechanism to be designed.

(2) Generate constraint space from the freedom space using the rules mentioned above.

(3) Derive the subspaces of the constraint space according to the equivalence relation of line graphs, and the dimension of each subspace is the same as that of the constraint space.

(4) Decompose the subspace and assign to each limb, and the number of limbs is not less than that of DOFs of the mechanism to be designed; actually, they are the same in most cases.

(5) Generate the corresponding freedom spaces for each limb from the constraint subspaces derived in step (4) using the dual rule.

(6) Configure the kinematic joints of each limb based on the corresponding freedom space, and generate the topological configurations of all limbs.

(7) Check the continuity of the movements; the mobility of the synthesized mechanism based on this method is instantaneous. Therefore, this step is very important to make sure of the functional effectiveness of the derived mechanism.

(8) if the generated mechanism does not have continuous movements, the synthesis stage should go back to step (6). If the generated mechanism has continuous movements, the type synthesis based on this subspace is finished, and another subspace should be selected, and the type synthesis should be carried out similarly.

The whole type synthesis process is presented in Figure 1.

2.3. Type Synthesis of $1 T 2 R$ Parallel Mechanisms. For the 1T2R type, the translational direction can be vertical or parallel to the plane composed of the rotational axes in initial position. Therefore, the type synthesis of mechanisms with 1T2R should be discussed separately. This paper focuses on the vertical situation, and the results for the parallel situation will be presented directly. The configurations of three chains and an active prismatic joint in each chain are used in the type synthesis of this paper.

For the vertical situation, the freedom-space atlas can be generated as shown in Figure 2(a). According to the Blanding rules, the constraint-space atlas can be generated as shown in Figure 2(b). This atlas represents two-dimensional planar forces and a couple with the axis perpendicular to the plane. 


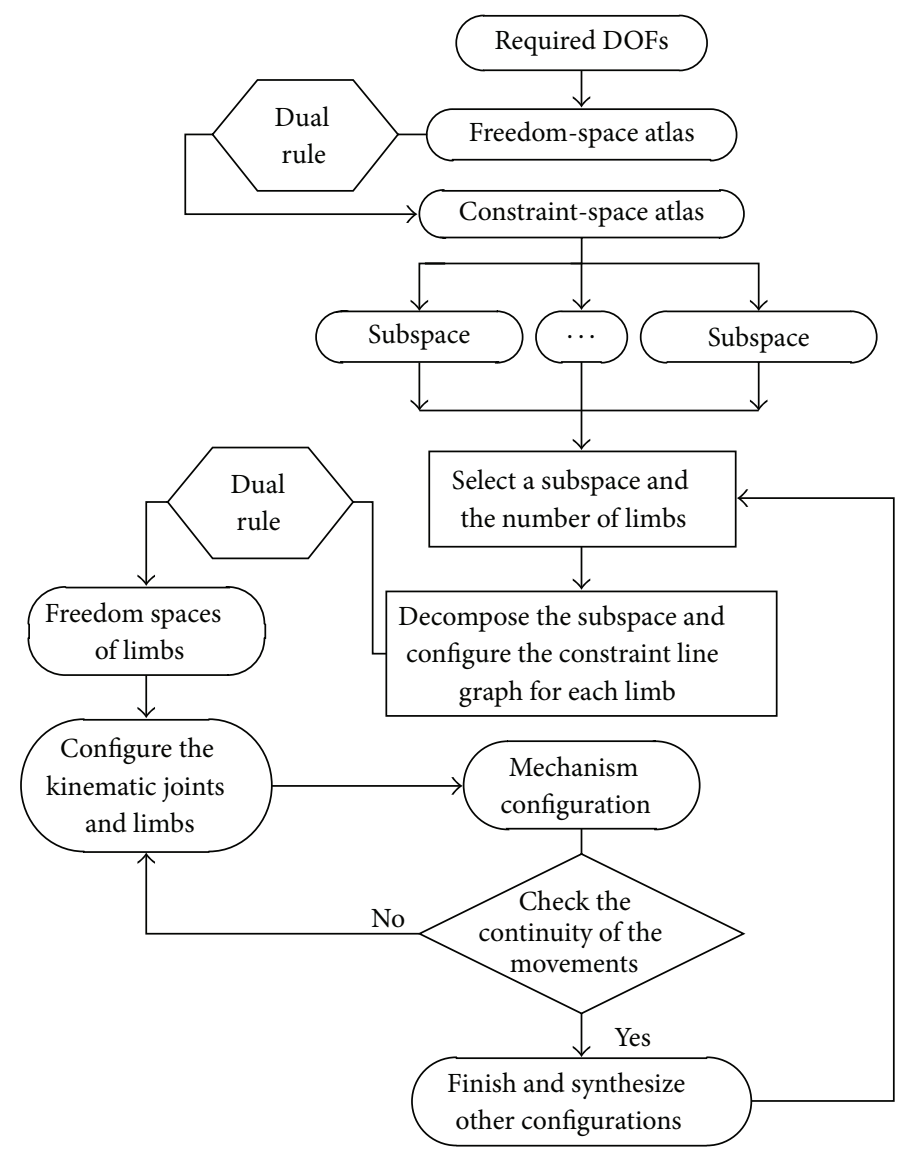

Figure 1: Type synthesis process based on Grassmann Line Geometry and Atlas Method.

(a)

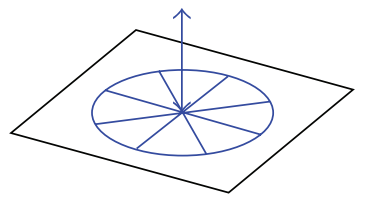

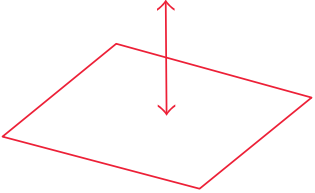

(b)

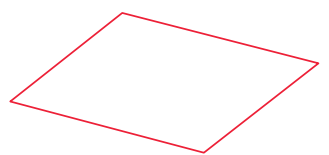

(c)

FIgURE 2: Atlases of freedom and constraint space: (a) freedom space; (b) constraint space I; (c) constraint space II.

Figure 2(c) is an equivalent atlas of that shown in Figure 2(b) according to the criterions of Grassmann Line Geometry.

Using the equivalence principle, some typical subspaces of the constraint space shown in Figure 2(b) can be derived and presented in Figure 3. According to the type synthesis process given in Figure 1, each subspace should be analyzed individually.

For the atlas presented in Figure 3(a), three onedimensional constraint spaces can be generated by decomposing the constraint space and being assigned to the limbs.

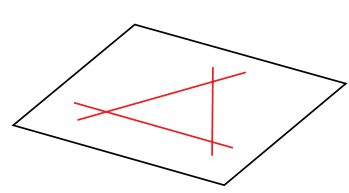

(a)

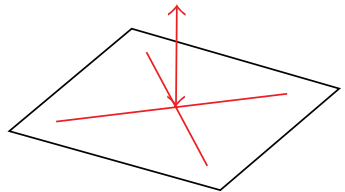

(b)
FIGURE 3: Subspaces with the same dimension: (a) subspace I; (b) subspace II.

Then, there is one-dimensional force constraint for each limb. According to the Blanding rules, the freedom space of each limb is composed of two-dimensional translations and three-dimensional rotations. Such a freedom space can be realized by a PRS (P: prismatic joint; R: revolute joint; S: spherical joint) limb. The three limbs can constitute a 3PRS mechanism, which is a typical lower-mobility parallel mechanism and has been extensively investigated in the field. It is well known that this mechanism has continuous movements and nonsingular workspace. The type synthesis process and results are presented in Table 4 .

For the atlas presented in Figure 3(b), the constraint spaces of the limbs can be assigned as follows: a threedimensional constraint space, that is, two-dimensional force constraints and one-dimensional couple constraint, 
TABLE 4: Type synthesis based on the subspace presented in Figure 3(a).

\begin{tabular}{|c|c|c|c|}
\hline & $\begin{array}{l}\text { Constraint-space atlas of } \\
\text { each limb }\end{array}$ & Freedom-space atlas & $\begin{array}{l}\text { Model and kinematic } \\
\text { joints of each limb }\end{array}$ \\
\hline \multicolumn{4}{|l|}{ 1st limb } \\
\hline & $\begin{array}{c}\text { One-dimensional force } \\
\text { constraint }\end{array}$ & $\begin{array}{l}\text { Two-dimensional translations and } \\
\text { three-dimensional rotations }\end{array}$ & PRS limb \\
\hline \multicolumn{4}{|l|}{ 2nd limb } \\
\hline & $\begin{array}{c}\text { One-dimensional force } \\
\text { constraint }\end{array}$ & $\begin{array}{l}\text { Two-dimensional translations and } \\
\text { three-dimensional rotations }\end{array}$ & PRS limb \\
\hline \multicolumn{4}{|l|}{ 3rd limb } \\
\hline & & $\begin{array}{l}\text { Two-dimensional translations and } \\
\text { three-dimensional rotations }\end{array}$ & PRS limb \\
\hline \multicolumn{4}{|l|}{ Synthesis result } \\
\hline & & $\begin{array}{l}\text { One translational DOF } \\
\text { and two rotational DOFs }\end{array}$ & \\
\hline
\end{tabular}

is assigned to the first limb; a two-dimensional constraint space, that is, one-dimensional force constraint and one-dimensional couple constraint, is assigned to the second limb; no constraint is assigned to the third limb. Then, the freedom space of the first limb is composed of onedimensional translation and two-dimensional rotations, and it can be realized by a PRR limb; the freedom space of the second limb is composed of two-dimensional translations and two-dimensional rotations, and it can be realized by a PRU (U: universal joint) limb; the freedom space of the third limb is a six-dimensional space and composed of threedimensional translations and three-dimensional rotations; such a freedom space can be realized by a PSU limb. The three limbs can constitute a virtual center mechanism (VCM). The continuity of movements and workspace of this mechanism have been discussed and confirmed in [35]. The detailed type synthesis process of this mechanism is presented in Table 5 .

When the constraint space is decomposed in a different way, a different mechanism can be generated on the basis of the type synthesis process presented above. The results are given directly in Table 6 . The parallel situation is also concluded in this table, and some typical mechanisms are listed. All of the mechanisms presented in this table are analyzed and confirmed to have continuous movements and workspace.

Note that the mechanisms given in Table 6 are just some synthesis results under typical situations; that is, the results presented in this paper are not exhaustive.

From the synthesis process presented above, it can be concluded that the type synthesis method used here is intuitive 
TABle 5: Type synthesis based on the subspace presented in Figure 3(b).

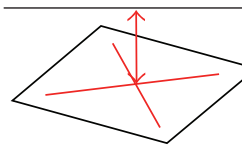

1 st limb

2nd limb

3rd limb
Freedom-space atlas

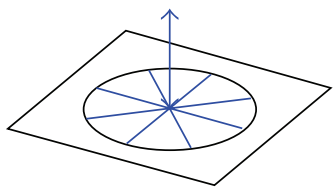

One-dimensional translation and two-dimensional rotations

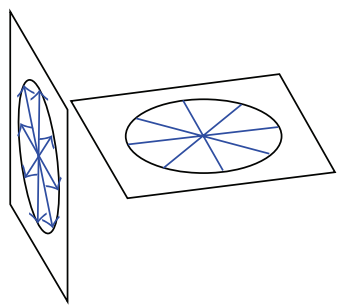

Two-dimensional translations and two-dimensional rotations

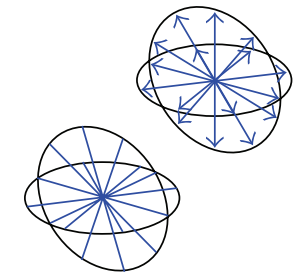

Three-dimensional translations and three-dimensional rotations

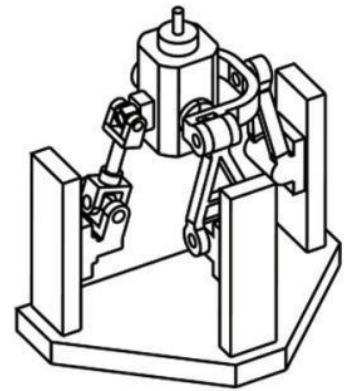

One translational DOF and two rotational DOFs

Synthesis result

ФNo constraint

One-dimensional force constraints and one-dimensional couple constraint
Model and kinematic joints of each limb

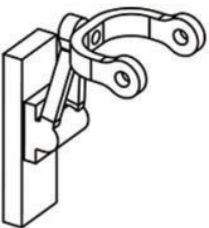

PRR limb

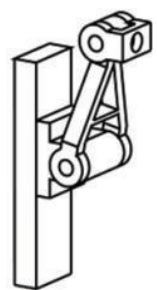

PRU limb

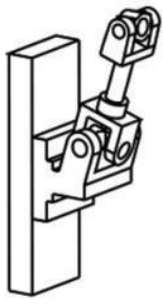

PSU limb and concise, thanks to the introduction of Grassmann Line Geometry and the use of atlas method. Combining the constraints and motions of mechanisms, this method also has clear and definite physical meaning. Therefore, this synthesis method should have good prospects in practical application.

\section{Typical Application}

The DS-Technologie has developed a series of Ecospeed machine centers (see Figure 4(a)) based on the Sprint Z3 tool head (see Figure 4(b)), and these machines have been successfully applied in industry, especially in the machining of structural aircraft parts and parts with freeform surfaces. The Sprint Z3 tool head is based on the 3-PRS parallel mechanism. Similarly, Fatronik developed a Space-5H machine center (see Figure 5(a)) based on a 3-DOF Hermes tool head (see Figure 5(b)). The DOF styles of Sprint Z3 and Hermes are $1 \mathrm{~T} 2 \mathrm{R}$, that is, a translational $\mathrm{DOF}$ and two rotational DOFs. The advantage of 1T2R lower mobility PKMs has been confirmed in practice. Note that both of the tool heads have 
TABLE 6: Type synthesis results of 1T2R parallel mechanisms.

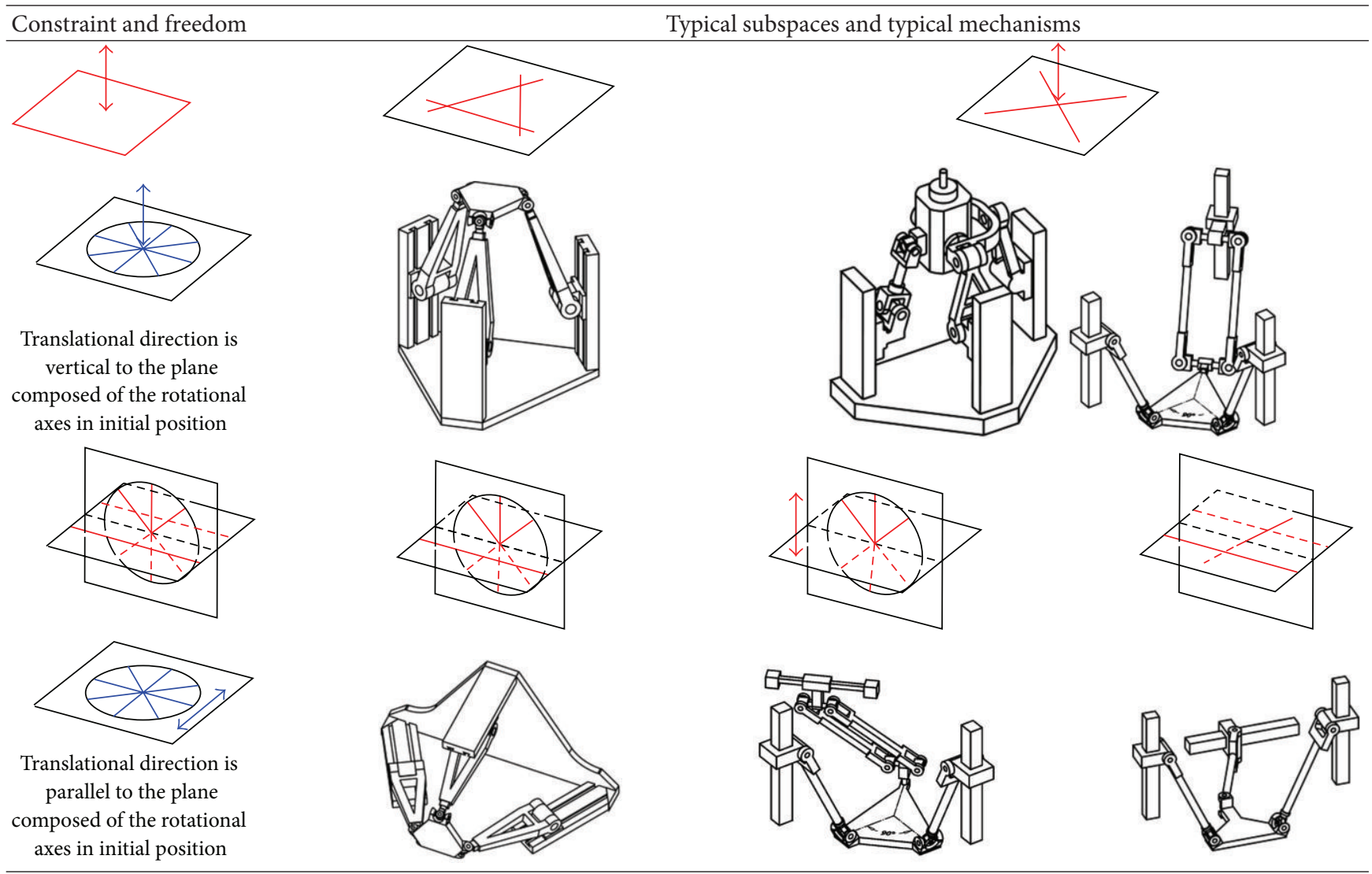

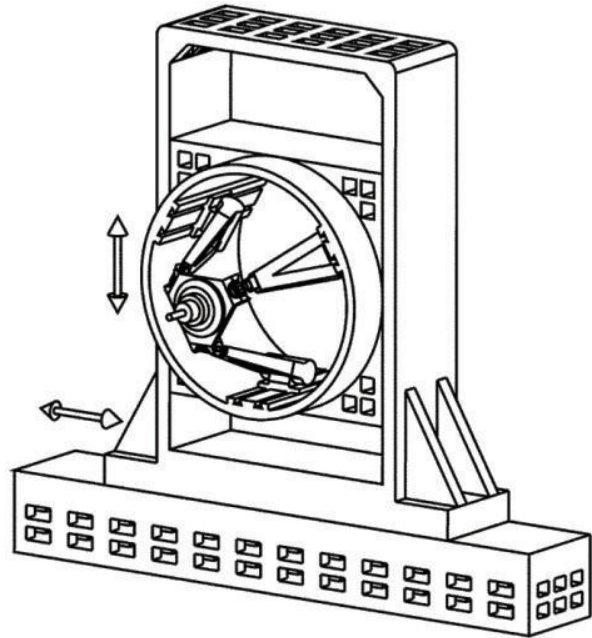

(a)

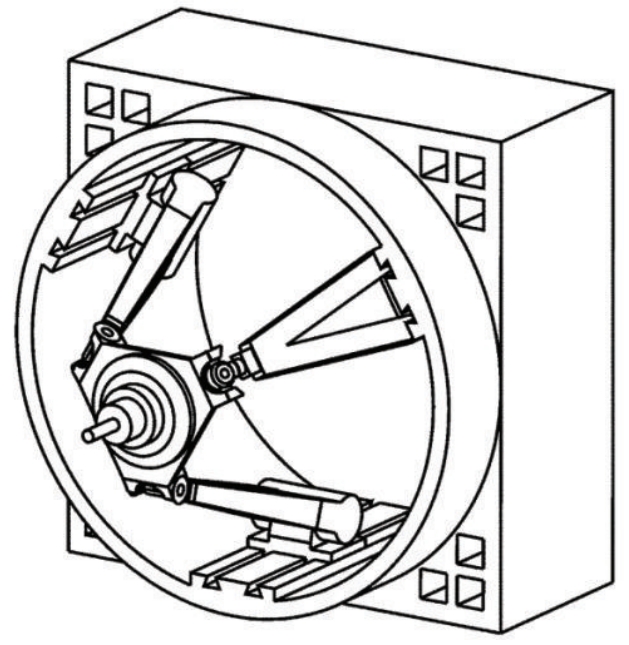

(b)

FIgure 4: CAD model: (a) Ecospeed machine center; (b) Sprint Z3 tool head.

parasitic motions [36]. It is well known that parasitic motions are the movements that occurred in the constraint directions and are the unexpected movements that simultaneously occurred with the movements in the freedom directions. The parasitic motions deteriorate the positioning accuracy and manipulability quality of a mechanism and increase the difficulty in control and kinematic calibration.

Since the parasitic motions are detrimental to the improvement of accuracy, 1T2R parallel mechanisms without parasitic motions will be welcome in the field. In Table 5, 


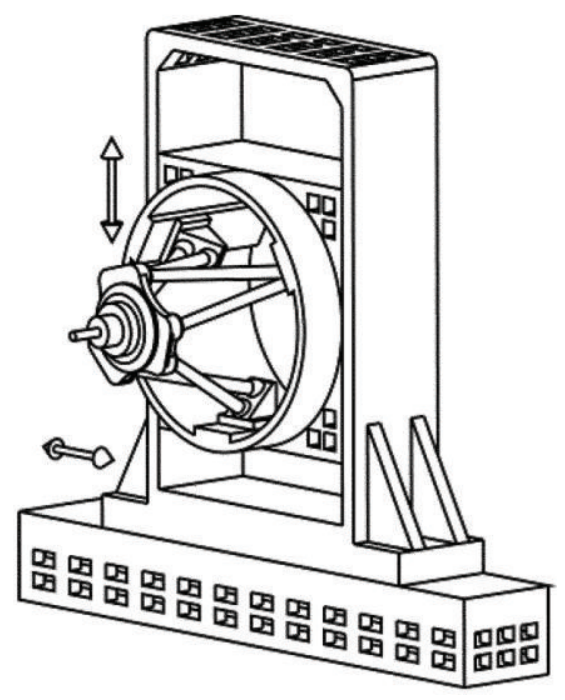

(a)

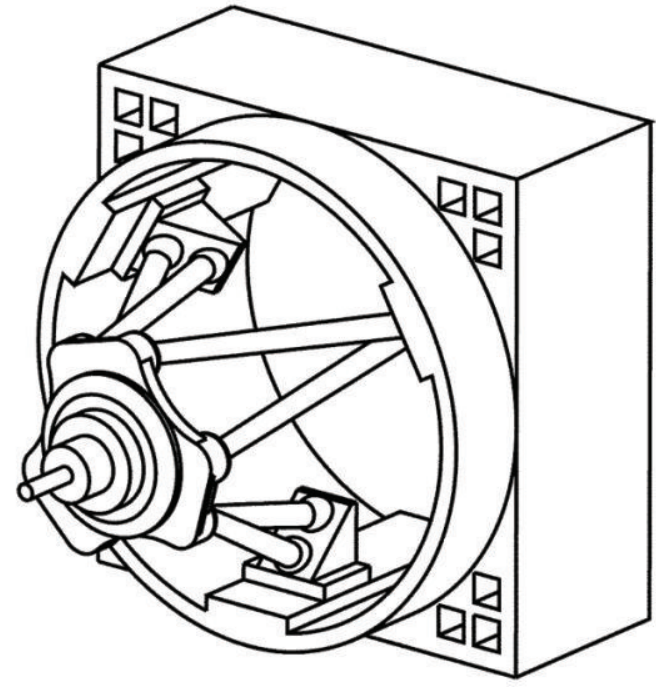

(b)

FIGURE 5: CAD model: (a) Space-5H machine center; (b) Hermes tool head.

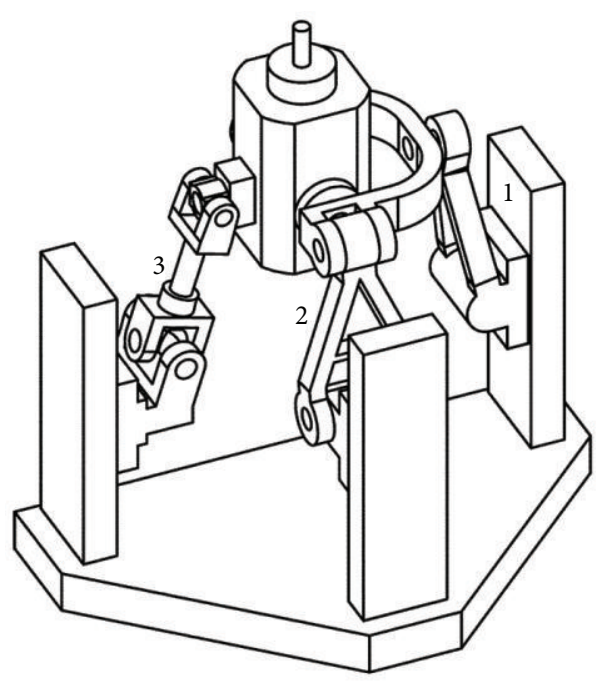

(a)

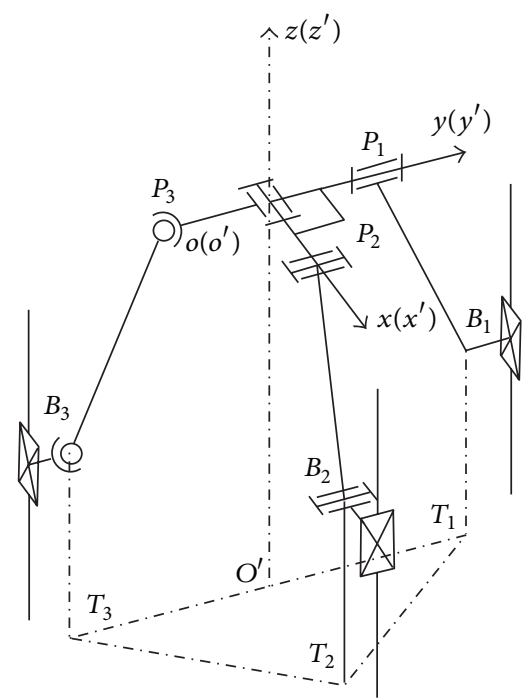

(b)

Figure 6: The 1T2R VCM: (a) CAD model; (b) kinematic scheme.

a $1 T 2 R$ VCM is derived; the CAD model and kinematic scheme are presented in Figure 6.

As shown in Figure 6(a), the mobile platform is connected to the base through three limbs. The first limb is a PRR kinematic chain. The second limb has a common bracket with the first limb, and a PRR kinematic chain is connected to the bracket. The third limb is a PSU kinematic chain and exerts no constraint on the mobile platform. All of the prismatic joints in the three limbs are active. As shown in Figure 6(b), all of the input motions are along the $z$-axis. When the slider $B_{1}$ in the first limb is fixed, the mobile platform has two rotational DOFs about the $x$ - and $y$-axes. The two rotational axes intersect at the origin $o$; therefore, a virtual center exists and there is no parasitic motion for this mechanism. This is a great advantage compared with the Sprint Z3 tool head and the Hermes tool head. So, a prototype will be designed and developed based on this VCM in this paper.

To achieve the necessary machining flexibility and efficiency in processing the parts with complicate and freeform surfaces, 5-DOF mechanism configurations are indispensable, such as the Ecospeed and Space-5H. Both of them are based on 5-DOF hybrid mechanisms and have three translational DOFs and two rotational DOFs (3T2R).

To constitute a 5-axis (3T2R) hybrid mechanism configuration based on the 3-DOF parallel mechanism proposed in Figure 6, another two translational DOFs are used. The directions of the two translational DOFs are perpendicular to each other and perpendicular to the input directions of 


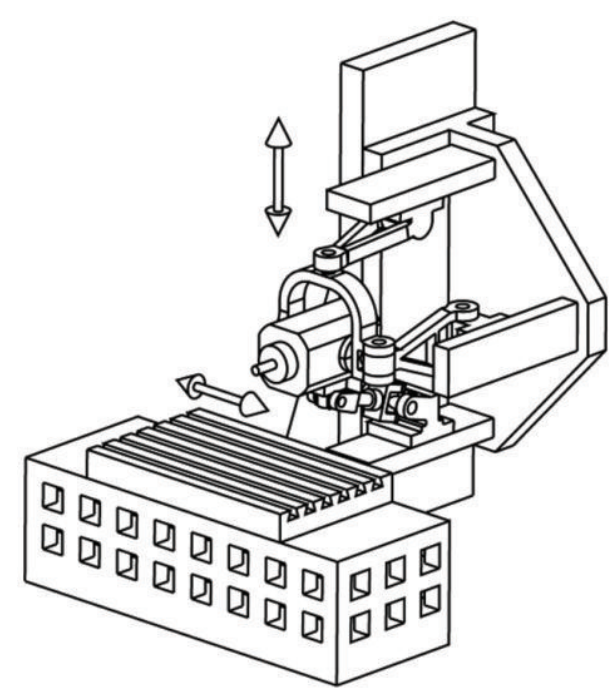

FIgure 7: The CAD model of the 5-axis (3T2R) mechanism.

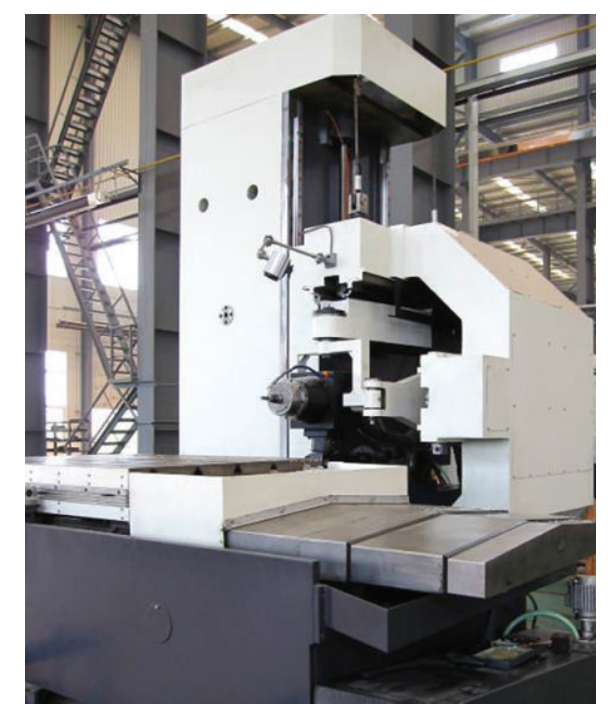

FIGURE 8: The developed 5-axis hybrid prototype.

the parallel mechanism. The CAD model of the 5-axis hybrid mechanism is presented in Figure 7.

The optimum kinematic design of the VCM has been discussed in [35]. Based on the optimization results and other relevant work, a prototype based on the 5-axis mechanism given in Figure 7 has been developed as shown in Figure 8. For the space limitation of this paper, the development process will not be presented in detail. The milling application using this prototype is carried out (see Figure 9), and the finished part is presented in Figure 10.

\section{Conclusion}

This paper introduced a systematic type synthesis method for lower mobility PKMs. The Grassmann Line Geometry was used as the mathematic foundation. On this basis, the atlas method was brought in to describe the motion or constraint

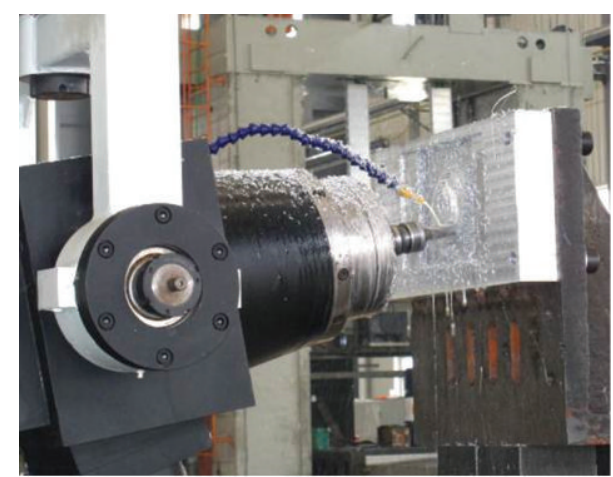

FIGURE 9: The machining process using the developed prototype.

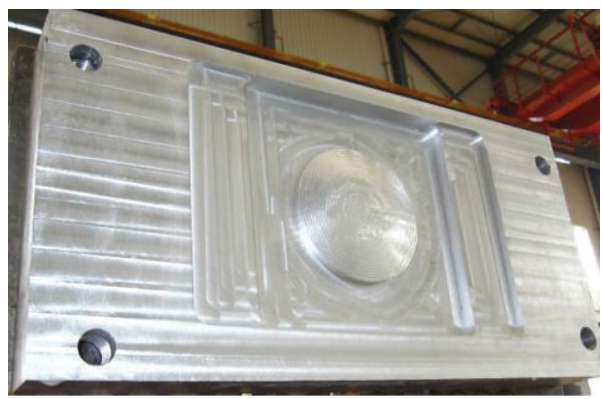

FIGURE 10: The part processed with the 5-axis hybrid prototype.

of a mechanism using freedom- or constraint-space line graphs. To identify the dimensions of the corresponding line clusters, some criterions of Grassmann Line Geometry were summarized. To investigate the relationship between the freedom and constraint of a mechanism, the Blanding rules and generalized Blanding rules were introduced sequentially. Using these rules, the mutual conversion between freedomspace atlas and constraint-space atlas could be realized. Thereafter, the technological process of the type synthesis was presented. The type synthesis of 1T2R PKMs based on the introduced method was carried out and the typical synthesis results were listed. Selected from the results, a VCM was used as the parallel module in the design of a 5-axis hybrid mechanism. A prototype based on the proposed hybrid mechanism was developed and the milling experiments were carried out and presented.

\section{Acknowledgments}

This work was supported in part by the National Natural Science Foundation of China under Grants 51135008 and 51305222, National Basic Research Program (973 Program) of China under Grant 2013CB035400, and China Postdoctoral Science Foundation under Grants 2012M520256 and 2013 T60107.

\section{References}

[1] B. Zi, J. Cao, Z. Zhu, and P. Mitrouchev, "Design, dynamics, and workspace of a hybrid-driven-based cable parallel manipulator," 
Mathematical Problems in Engineering, vol. 2013, Article ID 914653, 15 pages, 2013.

[2] C. B. Guo, K. R. Hao, and Y. S. Ding, "Neuroendocrinebased cooperative intelligent control system for multiobjective integrated control of a parallel manipulator," Mathematical Problems in Engineering, vol. 2012, Article ID 467402, 17 pages, 2012.

[3] Y. Zhang, W. A. Gruver, and F. Gao, "Dynamic simplification of three degree of freedom manipulators with closed chains," Robotics and Autonomous Systems, vol. 28, no. 4, pp. 261-269, 1999.

[4] S. Staicu, "Dynamics analysis of the Star parallel manipulator," Robotics and Autonomous Systems, vol. 57, no. 11, pp. 1057-1064, 2009.

[5] N. Shvalb, M. Shoham, H. Bamberger, and D. Blanc, "Topological and kinematic singularities for a class of parallel mechanisms," Mathematical Problems in Engineering, vol. 2009, Article ID 249349, 12 pages, 2009.

[6] A. Zubizarreta, M. Marcos, I. Cabanes, and C. Pinto, "A procedure to evaluate extended computed torque control configurations in the Stewart-Gough platform," Robotics and Autonomous Systems, vol. 59, no. 10, pp. 770-781, 2011.

[7] M. Geldart, P. Webb, H. Larsson, M. Backstrom, N. Gindy, and K. Rask, "A direct comparison of the machining performance of a variax 5 axis parallel kinetic machining centre with conventional 3 and 5 axis machine tools," International Journal of Machine Tools and Manufacture, vol. 43, no. 11, pp. 1107-1116, 2003.

[8] K. H. Harib, A. M. M. Sharif Ullah, and A. Hammami, "A hexapod-based machine tool with hybrid structure: kinematic analysis and trajectory planning," International Journal of Machine Tools and Manufacture, vol. 47, no. 9, pp. 1426-1432, 2007.

[9] P. Pham, Design of Hybrid-Kinematic Mechanisms for Machine Tools, École Polytechnique Fédérale de Lausanne, Lausanne, Switzerland, 2009.

[10] D. Kanaan, P. Wenger, and D. Chablat, "Kinematic analysis of a serial-parallel machine tool: the VERNE machine," Mechanism and Machine Theory, vol. 44, no. 2, pp. 487-498, 2009.

[11] Q. C. Li and J. M. Hervé, "1T2R parallel mechanisms without parasitic motion," IEEE Transactions on Robotics, vol. 26, no. 3, pp. 401-410, 2010.

[12] Q. Chen, Z. Chen, X. Chai, and Q. Li, "Kinematic analysis of a 3-axis parallel manipulator the P3," Advances in Mechanical Engineering, vol. 2013, Article ID 589156, 10 pages, 2013.

[13] C. C. Kao and T. S. Zhan, "Modified PSO method for robust control of 3RPS parallel manipulators," Mathematical Problems in Engineering, vol. 2010, Article ID 302430, 25 pages, 2010.

[14] X. Liu, F. Xie, L. Wang, and J. Wang, "Optimal design and development of a decoupled A/B-axis tool head with parallel kinematics," Advances in Mechanical Engineering, vol. 2010, Article ID 474602, 14 pages, 2010.

[15] J. J. Yu, J. S. Dai, S. Bi, and G. Zong, “Type synthesis of a class of spatial lower-mobility parallel mechanisms with orthogonal arrangement based on Lie group enumeration," Science China Technological Sciences, vol. 53, no. 2, pp. 388-404, 2010.

[16] Y. Lu, Y. Lu, N. Ye, B. Mao, J. Han, and C. Sui, "Derivation of valid contracted graphs from simpler contracted graphs for type synthesis of closed mechanisms," Mechanism and Machine Theory, vol. 52, pp. 206-218, 2012.
[17] Y. Lu, L. Ding, and J. Yu, "Autoderivation of topological graphs for type synthesis of planar 3DOF parallel mechanisms," Journal of Mechanisms and Robotics, vol. 2, no. 1, pp. 1-8, 2010.

[18] Z. Huang and Q. C. Li, "General methodology for type synthesis of symmetrical lower-mobility parallel manipulators and several novel manipulators," International Journal of Robotics Research, vol. 21, no. 2, pp. 131-145, 2002.

[19] X. W. Kong and C. M. Gosselin, "Type synthesis of 3-DOF spherical parallel manipulators based on screw theory," Journal of Mechanical Design, vol. 126, no. 1, pp. 101-108, 2004.

[20] Y. F. Fang and L. W. Tsai, "Structure synthesis of a class of 4-DoF and 5-DoF parallel manipulators with identical limb structures," International Journal of Robotics Research, vol. 21, no. 9, pp. 799810, 2002.

[21] S. Guo, Y. Fang, and H. Qu, "Type synthesis of 4-DOF nonoverconstrained parallel mechanisms based on screw theory," Robotica, vol. 30, no. 1, pp. 31-37, 2012.

[22] Q. Zeng and Y. F. Fang, "Structural synthesis and analysis of serial-parallel hybrid mechanisms with spatial multi-loop kinematic chains," Mechanism and Machine Theory, vol. 49, pp. 198-215, 2012.

[23] J. M. Hervé, "Lie group of rigid body displacements, a fundamental tool for mechanism design," Mechanism and Machine Theory, vol. 34, no. 5, pp. 719-730, 1999.

[24] Q. C. Li, Z. Huang, and J. M. Hervé, “Type synthesis of 3R2T 5DOF parallel mechanisms using the lie group of displacements," IEEE Transactions on Robotics and Automation, vol. 20, no. 2, pp. 173-180, 2004.

[25] J. Meng, G. F. Liu, and Z. X. Li, "A geometric theory for analysis and synthesis of sub-6 DoF parallel manipulators," IEEE Transactions on Robotics, vol. 23, no. 4, pp. 625-649, 2007.

[26] T. L. Yang, A. X. Liu, Q. Jin, Y. F. Luo, H. P. Shen, and L. B. Hang, "Position and orientation characteristic equation for topological design of robot mechanisms," Journal of Mechanical Design, vol. 131, no. 2, pp. 0210011-02100117, 2009.

[27] Q. Jin and T. L. Yang, "Theory for topology synthesis of parallel manipulators and its application to three-dimensiontranslation parallel manipulators," Journal of Mechanical Design, vol. 126, no. 4, pp. 625-639, 2004.

[28] F. Gao, J. Yang, and Q. J. Ge, “Type synthesis of parallel mechanisms having the second class GF sets and two dimensional rotations," Journal of Mechanisms and Robotics, vol. 3, no. 1, Article ID 011003, 2010.

[29] B. Motevalli, H. Zohoor, and S. Sohrabpour, "Structural synthesis of 5 DoFs 3T2R parallel manipulators with prismatic actuators on the base," Robotics and Autonomous Systems, vol. 58 , no. 3, pp. 307-321, 2010.

[30] J. P. Merlet, "Singular configurations of parallel manipulators and Grassmann geometry," International Journal of Robotics Research, vol. 8, no. 5, pp. 45-56, 1989.

[31] B. Monsarrat and C. M. Gosselin, "Singularity analysis of a three-leg six-degree-of-freedom parallel platform mechanism based on grassmann line geometry," International Journal of Robotics Research, vol. 20, no. 4, pp. 312-326, 2001.

[32] A. Wolf and D. Glozman, "Singularity analysis of large workspace 3RRRS parallel mechanism using line geometry and linear complex approximation," Journal of Mechanisms and Robotics, vol. 3, no. 1, Article ID 011004, 2010.

[33] J. J. Yu, S. Z. Li, H. J. Su, and M. L. Culpepper, "Screw theory based methodology for the deterministic type synthesis of flexure mechanisms," Journal of Mechanisms and Robotics, vol. 3, no. 3, Article ID 031008, 2011. 
[34] J. J. Yu, S. Z. Li, X. Pei, S. S. Bi, and G. Zong, "A unified approach to type synthesis of both rigid and flexure parallel mechanisms," Science China Technological Sciences, vol. 54, no. 5, pp. 12061219, 2011.

[35] F. Xie, X. Liu, and J. Wang, "A 3-DOF parallel manufacturing module and its kinematic optimization," Robotics and Computer, vol. 28, no. 3, pp. 334-343, 2012.

[36] X. J. Liu, L. P. Wang, F. G. Xie, and I. A. Bonev, "Design of a three-axis articulated tool head with parallel kinematics achieving desired motion/force transmission characteristics," Journal of Manufacturing Science and Engineering, vol. 132, no. 2, pp. 0210091-0210098, 2010. 


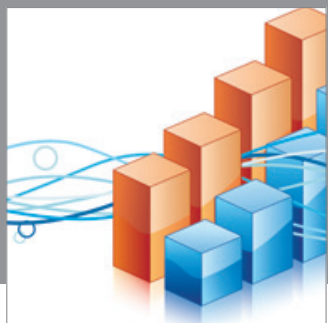

Advances in

Operations Research

mansans

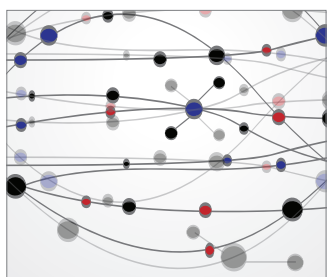

The Scientific World Journal
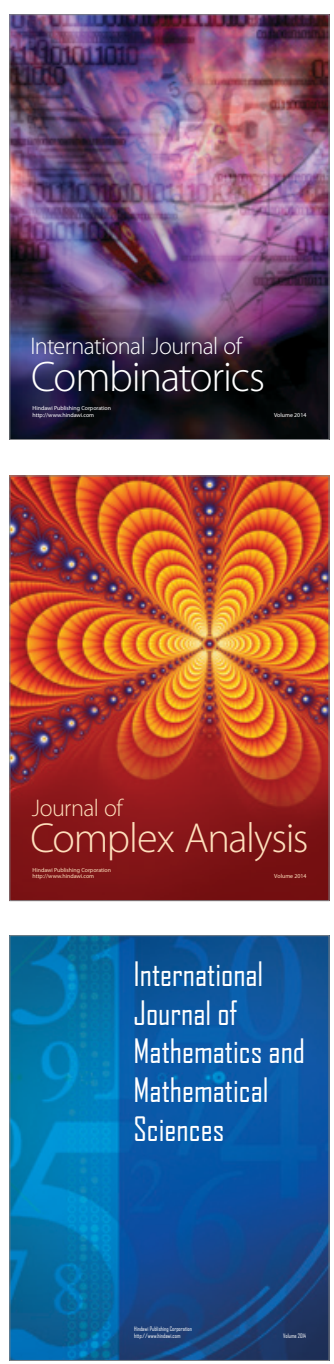
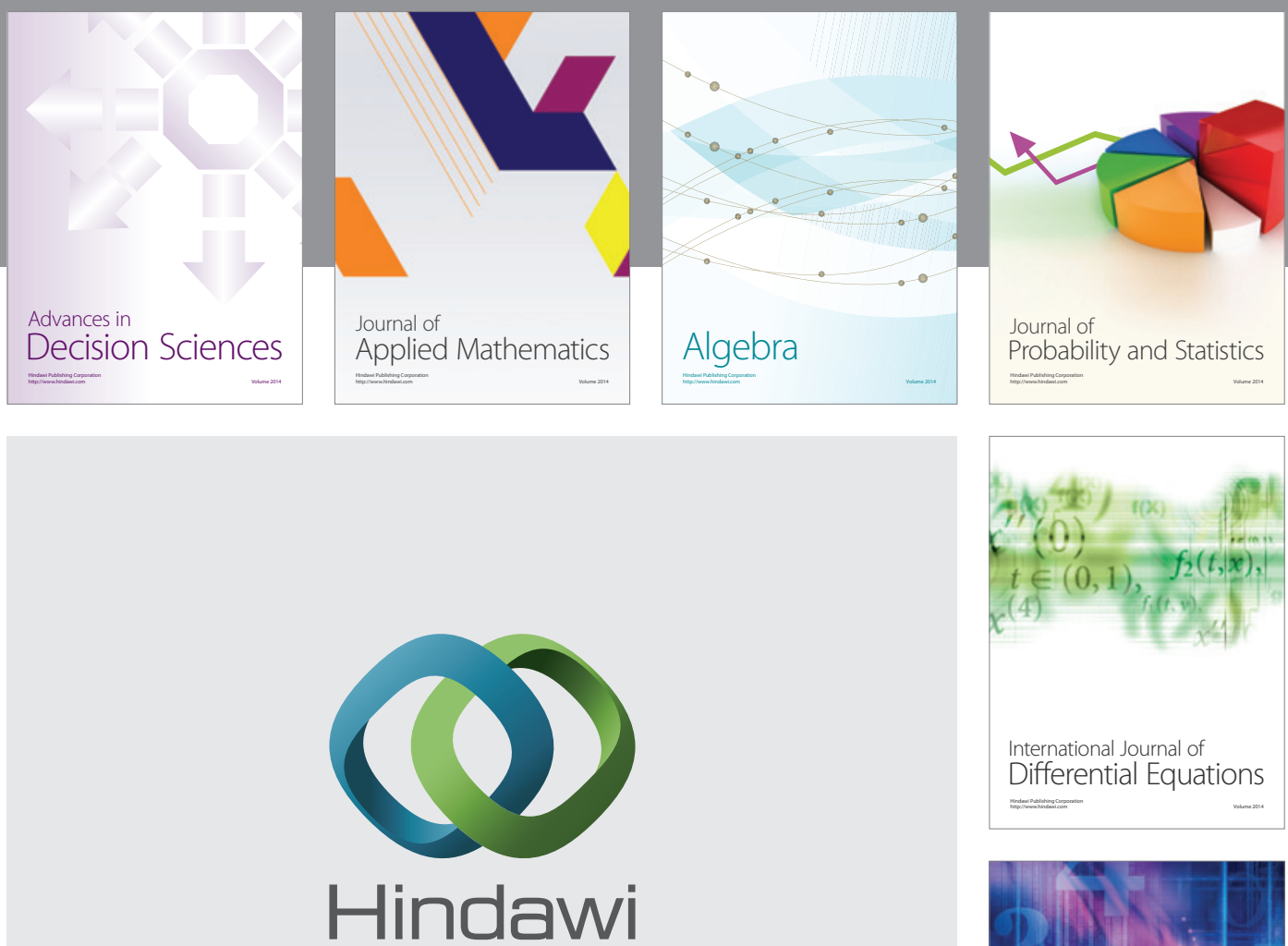

Submit your manuscripts at http://www.hindawi.com
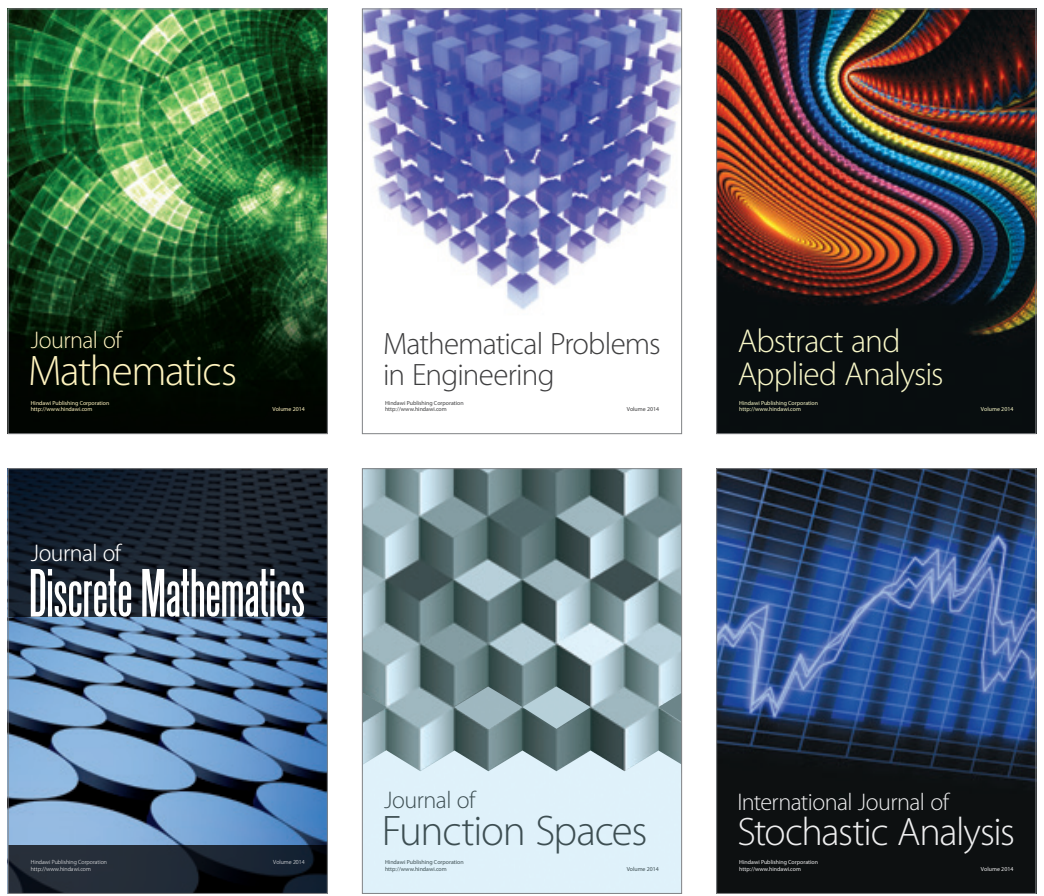

Journal of

Function Spaces

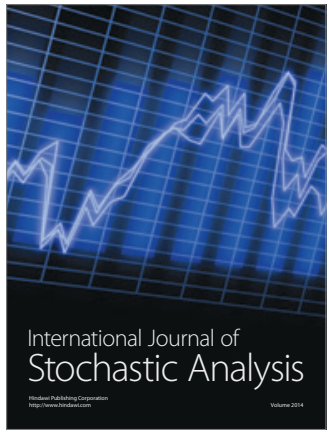

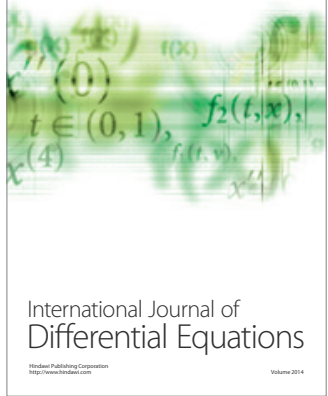
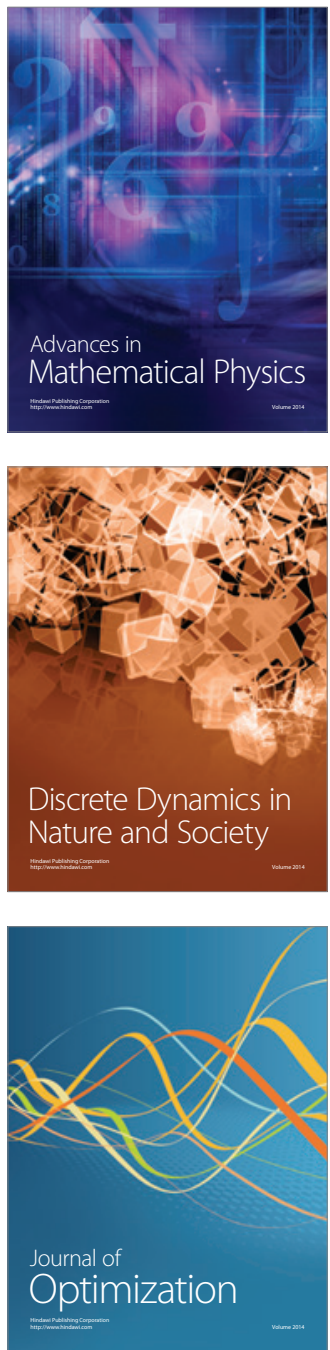